\title{
Uso de Resíduos na Agricultura
}

Fabio Olivieri de Nobile*

\section{Resumo}

Este artigo apresenta uma revisão dos resumos da XX Reunião Brasileira de Fertilidade do Solo e Nutrição de Plantas, realizada em Piracicaba-São Paulo. A utilização agronômica de resíduos pressupõe um número bem diverso de aplicações, entre as quais se podem lembrar seu uso em alimentação animal, substrato para fermentações, fabricação de fertilizantes orgânicos ou organominerais, cobertura de pisos ("camas") em diferentes criações e sucedâneos de matéria-prima para as agroindústrias ou atividades assemelhadas. Entretanto, o objetivo desta apresentação é restrito aos aspectos do uso de resíduos no solo, compreendendo os aspectos relacionados à caracterização desses resíduos, os benefícios ou inconvenientes de sua aplicação no solo e os parâmetros que devem ser observados quando se pretende dar esse destino aos resíduos. Dessa maneira, o assunto a ser tratado pode ser dividido em dois aspectos fundamentais: os resíduos e os solos. No tocante aos resíduos, os principais fatores que afetam sua aplicação no solo são: composição química, características físicas, aspectos sanitários, quantidade gerada e regime de liberação. Já em relação ao solo, devem-se considerar prioritariamente todas aquelas características responsáveis pela capacidade do solo em desativar e estabilizar os resíduos, por meio de mecanismos físicos, químicos e biológicos. É dentro desse quadro geral que se pretende abordar alguns aspectos específicos e considerados mais importantes para a utilização dos resíduos no solo.

Palavras-chave: Agricultura; Resíduos; Solo.

\section{Caracterização dos resíduos}

De acordo com Glória (2004), a caracterização adequada dos resíduos é básica para definir o seu uso no solo e o conhecimento da sua origem, e compreende:

$\checkmark$ Matéria-prima utilizada e suas características (quantidade, tipo, origem);

$\checkmark$ Produtos acrescentados ao processo (tipo, quantidade, etapa);

\footnotetext{
*Doutor em Agronomia, Unesp-Jaboticabal; docente do Centro Universitário de Araraquara - Uniara e do Centro Universitário da Fundação Educacional de Barretos - Unifeb. E-mail: fonobile@ yahoo.com.br.
} 
$\checkmark$ Regime de produção (contínuo, intermitente, sazonal);

$\checkmark$ Tipo(s), quantidade(s) e regime de vazão do(s) resíduos;

$\checkmark$ Aspectos do(s) resíduo(s) (estado físico, temperatura);

$\checkmark$ Pré-tratamentos.

Essa etapa possibilita estimar a variabilidade da composição do resíduo, facilita a caracterização química e já permite iniciar o direcionamento do estudo das possibilidades de uso do solo. É fundamental para que se estabeleça o chamado Plano de Amostragem do(s) Resíduo(s), processo básico para uma adequada avaliação da composição química. O Plano de Amostragem deve considerar (GLÓRIA, 2004):

$\checkmark$ Resíduos a serem amostrados;

$\checkmark$ Locais de amostragens;

$\checkmark$ Frequências, volume, número e tipo das amostras;

$\checkmark$ Tipo de amostradores;

$\checkmark$ Método de preservação e estocagem.

Algumas dessas etapas já podem ser conduzidas de acordo com a Associação Brasileira de Normas Técnicas (ABNT) e, especificamente, devem ser consultadas a NBR 9897/87 - que trata do planejamento de amostragens de efluentes líquidos - a NBR 9898/87 - que normatiza técnicas de amostrageme preservação de amostras de resíduos - e a NBR 1007/87 - sobre locais de amostragens, tipo de amostradores, condições e tempo de estocagem das amostras.

É bastante comum a inexistência dessas etapas nos estudos agronômicos sobre utilização de resíduos, o que torna os dados a respeito de sua composição química incompletos, por não permitir uma visão mais completa do processo de geração e das possibilidades de variação nas características químicas.

A avaliação química de um resíduo pode e deve ser conduzida em função das características prévias já levantadas; isso facilita e muitas vezes reduz o tipo e número de análises químicas a realizar. ANBR 9897 apresenta algumas recomendações de parâmetros mínimos para controle de efluentes líquidos, mas no geral pode considerarse os seguintes parâmetros:

$\checkmark$ Umidade;

$\checkmark$ Matéria orgânica;

$\checkmark \mathrm{pH}$;

$\checkmark$ Acidez ou alcalinidade;

$\checkmark$ Macronutrientes primários e secundários;

$\checkmark$ Micronutrientes;

$\checkmark$ Metais pesados, além daqueles considerados micronutrientes $(\mathrm{Pb}, \mathrm{Cr}, \mathrm{Hg}$, As, Cd); 
$\checkmark$ Sódio;

$\checkmark$ Condutividade;

$\checkmark$ Coliformes fecais;

$\checkmark$ Qualquer substância tóxica que se suspeite estar presente em função do processo de geração.

Deve-se considerar que o conhecimento adequado do processo de geração do resíduo permite excluir determinadas análises, visto não haver possibilidade de introdução do elemento ou substâncias no processo, e indica o potencial de nocividade de um resíduo pela presença de substâncias tóxicas.

É também o conhecimento do processo de geração que indicará a necessidade ou não de caracterizar a presença de determinadas formas de um elemento. Isso ocorre particularmente no caso do nitrogênio, fósforo, enxofre e substâncias orgânicas, pois em processos de fabricação há transformações, criando às vezes uma nocividade potencial do resíduo, a partir de uma situação original não preocupante.

\section{UTILIZAÇão do ResíduO No SOLO}

A partir da caracterização química do resíduo, pode-se estimar a possibilidade da sua utilização no solo, considerando:

$\checkmark$ Os preceitos legais existentes;

$\checkmark$ A avaliação dos efeitos no solo;

$\checkmark$ Taxa de aplicação;

$\checkmark$ Compatibilidade com a cobertura vegetal.

\section{Preceitos legais}

Em relação aos preceitos legais, é importante considerar o que dispõe o Título IV do regulamento da Lei 997, de 31/5/76, do governo do Estado de São Paulo, que dispõe sobre a prevenção e o controle da poluição do meio ambiente.

O exame da legislação é sempre uma etapa importante, porquanto facilitará não só a caracterização do resíduo, face aos órgãos de controle, como também mostrará eventuais entraves ao que se pretenda.

\section{Avaliação dos efeitos no solo}

Para Defelipo et al. (1992), uma adequada caracterização química permite razoável aproximação dos efeitos que a aplicação do resíduo pode causar ao solo. Entretanto, devido às diversas variáveis influenciando esses efeitos, muitas vezes somente a caracterização química não é suficiente, servindo apenas como guia da provável taxa de aplicação no solo. 
Isso significa que, sendo um resíduo cujos efeitos no solo e nas culturas não são ainda perfeitamente conhecidos, é necessário o estudo específico, adicionando-se doses do resíduo ao(s) solo(s) e aferindo-se os seus efeitos (PERUCCI, 1990).

No geral, os seguintes aspectos são considerados:

\section{pH}

Para Veloso et al. (1992), a influência da adição de um resíduo nas características de acidez ou alcalinidade dos solos é avaliada principalmente pelos seguintes parâmetros da caraterização química:

$\checkmark \mathrm{pH}$ do resíduo;

$\checkmark$ acidez ou alcalinidade;

$\checkmark$ teor de matéria orgânica;

$\checkmark$ presença de substâncias potencialmente ácidas ou alcalinas, quando reagem no solo.

A determinação do $\mathrm{pH}$ de um resíduo deve considerar o seu estado físico natural. Se líquido, o pH pode ser lido diretamente no resíduo. Se pastoso ou sólido, através do preparo de uma suspensão com água destilada (FALCÃO, 2005).

No caso de pastosos ou líquidos não há uma metodologia bem estabelecida, encontrando-se relações-resíduo: água variando de 2,5:1 até 50:1. Isso já serve para evidenciar que a medida do $\mathrm{pH}$ é apenas um indicador das características ácidas ou alcalinas do resíduo, sendo imprescindível que se determine o seu potencial de acidez ou alcalinidade, de acordo com determinação específica. No geral, isso é feito mediante a colocação do resíduo de características alcalinas em solução com excesso de ácido. Após um período, titula-se a acidez remanescente e, por diferença, a alcalinidade potencial do resíduo. No caso de resíduo ácidos, coloca-se uma amostra do material em excesso de solução alcalina e, após um período de tempo, determina-se a alcalinidade residual e, por diferença, a acidez potencial do resíduo (FALCÃO, 2005).

Entretanto, o fato de um resíduo ter determinadas características de acidez ou alcalinidade não significa que a sua incorporação no solo vai acidificá-lo ou alcalinizálo. Isso é quase certo nos casos de resíduos minerais; porém, nos resíduos com carga orgânica, nem sempre ocorre (FALCÃO, 2005).

Provavelmente o exemplo mais conhecido é o da vinhaça, proveniente da fermentação alcoólica. $\mathrm{O}$ pH desse resíduo é inferior a 7 (de 3,5 a 5,0, dependendo do tipo da vinhaça) e sua acidez potencial bastante variável, conforme se pode observar na Tabela 1.

Entretanto, a adição de vinhaça no solo provoca uma elevação do seu $\mathrm{pH}$, de acordo com exemplos bastante conhecidos. Na Tabela 2, são apresentados os 
resultados de adição de vinhaça no pH e alumínio "trocável" de três solos, que servem como exemplo geral do fenômeno.

Tabela 1. Valores de $\mathrm{pH}$ e da acidez de diferentes vinhaças

(RODELLA e FERRARI, 1977).

\begin{tabular}{cccc}
\hline AMOSTRAS & & ACIDEZ & pH \\
\hline & & e.g. $\mathrm{H}^{+} \mathrm{L}^{-1}$ & \\
4 & Us. da Barra & 0,775 & 4,40 \\
7 & Us. Santa Elisa & 0,151 & 4,00 \\
12 & Us. da Pedra & 0,142 & 4,20 \\
14 & Us. Barbacena & 0,186 & 3,80 \\
16 & Us. Costa Pinto & 0,215 & 4,10 \\
\hline
\end{tabular}

(1) Titulação com solução de $\mathrm{NaOH}$ até $\mathrm{pH}$ 7; e.g. $\mathrm{H}^{+} \mathrm{L}^{-1}$ (equivalente em gramas de $\mathrm{H}^{+}$ por litro)

Tabela 2. Efeito da adição de vinhaça e algumas características de acidez dos solos (dados extraídos de MATTIAZZO e GLÓRIA, 1985).

\begin{tabular}{|c|c|c|c|c|c|c|c|c|c|c|c|c|}
\hline \multirow{3}{*}{ Solo $^{(1)}$} & \multicolumn{12}{|c|}{ Dias após a aplicação de vinhaça } \\
\hline & \multicolumn{2}{|c|}{0} & \multicolumn{2}{|c|}{14} & \multicolumn{2}{|c|}{36} & \multicolumn{2}{|c|}{67} & \multicolumn{2}{|c|}{123} & \multicolumn{2}{|c|}{178} \\
\hline & $\mathrm{pH}$ & $\mathrm{Al}$ & $\mathrm{pH}$ & $\mathrm{Al}$ & $\mathrm{pH}$ & $\mathrm{Al}$ & $\mathrm{pH}$ & $\mathrm{Al}$ & $\mathrm{pH}$ & $\mathrm{Al}$ & $\mathrm{pH}$ & $\mathrm{Al}$ \\
\hline AQ (T) & 5,8 & 0,17 & 5,8 & 0,16 & 5,5 & 0,17 & 4,9 & 0,24 & 4,9 & 0,27 & 5,2 & 0,32 \\
\hline $\mathrm{AQ}(\mathrm{V})$ & 4,7 & 0,52 & 5,5 & 0,09 & 5,9 & 0,03 & 5,9 & 0 & 6,4 & 0 & 6,3 & 0 \\
\hline $\mathrm{LR}(\mathrm{T})$ & 6,4 & 0 & 6,5 & 0 & 6,4 & 0 & 6,3 & 0 & 6,2 & 0 & 5,6 & 0 \\
\hline $\mathrm{LR}(\mathrm{V})$ & 5,7 & 0 & 6,1 & 0 & 6,2 & 0 & 6,1 & 0 & 6,3 & 0 & 5,8 & 0 \\
\hline PVA (T) & 5,5 & 0,81 & 5,3 & 0,87 & 5,4 & 0,84 & 5,2 & 0,54 & 4,6 & 0,87 & 4,5 & 0,89 \\
\hline PVA (V) & 4,5 & 0,77 & 4,7 & 0,64 & 4,9 & 0,43 & 4,9 & 0,43 & 5,2 & 0,42 & 5,8 & 0,33 \\
\hline
\end{tabular}

(I) (T) Testemunha, (V) solo com vinhaça na base de $500 \mathrm{~m}^{3}$ ha ${ }^{-1}$; AQ (areia quartzosa); LR (Latossolo Roxo); PVA (Argissolo Vermelho Amarelo)

Exemplos similares a esse, com outros resíduos orgânicos, são conhecidos. Na Figura 1 é representada a variação no $\mathrm{pH}$ do solo submetido a tratamento com o licor negro da fabricação do furfural, com neutralização total $(100 \% \mathrm{CaO})$, parcial $(50 \% \mathrm{CaO})$ e sem neutralização, pelo hidróxido de cálcio. O licor negro de fabricação do furfural tem $\mathrm{pH}=2,0$ e acidez de 0,35 e.g $\mathrm{H}^{+} \mathrm{kg}^{-1}, \operatorname{com} 2,8 \%$ de matéria orgânica.

Esse efeito decorre da carga orgânica desses resíduos, que sofre uma oxidação no solo, provocando a liberação de elétrons que são recebidos pelo $\mathrm{O}^{2}$ ou $\mathrm{H}^{+}$, resultando na neutralização direta da acidez (no caso do $\mathrm{H}^{+}$) ou indireta, por meio da formação do íon oxigênio. Portanto, quanto maior a carga orgânica recebida pelo solo, mais evidente é o fenômeno. 


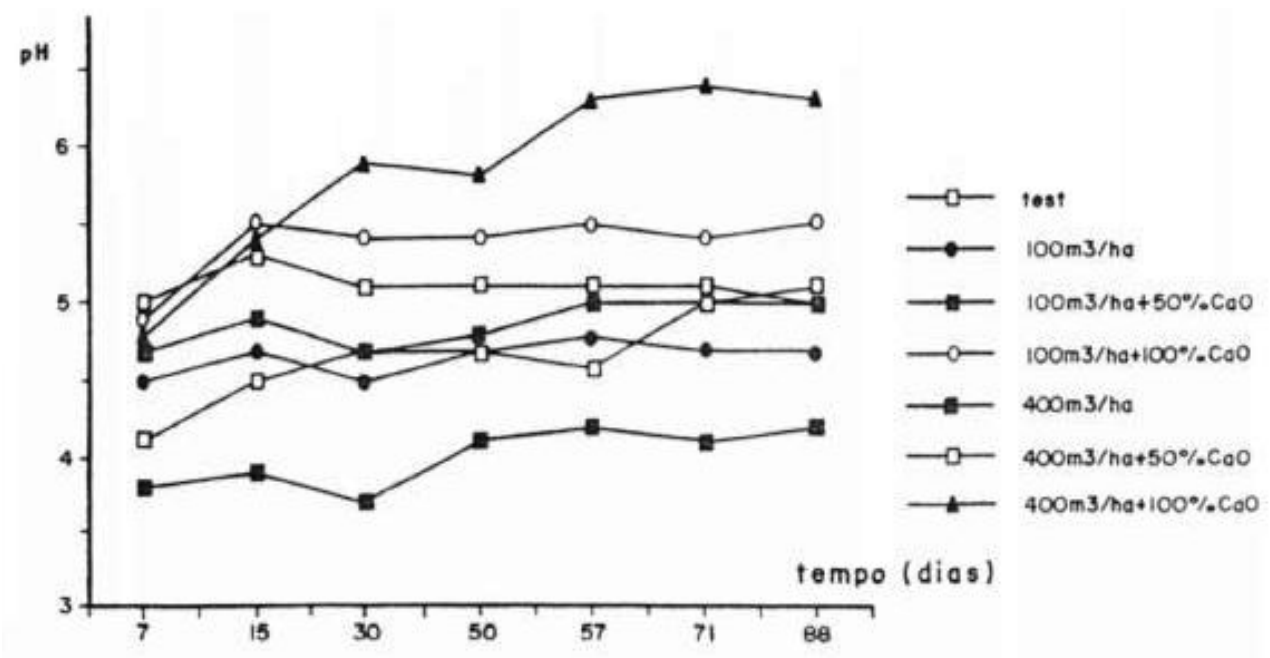

Figura 1. Variação no $\mathrm{pH}$ do solo nos tratamentos com "licor negro" natural e neutralizado com CaO. (GLÓRIAe MATIAZZO, 1991).

Componentes eventuais dos resíduos podem provocar elevação da acidez do solo, sem que o $\mathrm{pH}$ do resíduo caracterize exatamente esse potencial. Um exemplo típico é o de resíduos contendo enxofre ou óxidos derivados $\left(\mathrm{SO}_{2}\right)$, que podem causar grande aumento de acidez sem, no entanto, ter-se $\mathrm{pH}$ baixo no resíduo (OSÓRIO FILHO, 2006).

A importância de conhecer-se o efeito da adição dos resíduos no $\mathrm{pH}$ do solo é evidente, tanto pelos efeitos diretos que podem causar nos vegetais, como pelos efeitos indiretos na disponibilidade de nutrientes e de metais pesados.

\section{Capacidade de troca catiônica (CTC)}

Para Glória (2004), os resíduos contendo matéria orgânica podem contribuir para a elevação da CTC do solo. Entretanto, esse efeito varia bastante com:

$\checkmark$ teor de material orgânico do resíduo;

$\checkmark$ tipo de compostos orgânicos predominantes no resíduo;

$\checkmark$ velocidade de oxidação desse material orgânico no solo;

$\checkmark$ quantidade do resíduo aplicado.

Muitos resíduos apresentam o carbono orgânico em formas simples - açúcares, amido, ácidos orgânicos -, outros, em formas mais complexas - celulose, lignina ou óleos -, e, muitas vezes, misturas em proporções variadas desses componentes orgânicos (GLÓRIA, 2004).

A velocidade de decomposição desses resíduos no solo, no sentido da formação 
do húmus, ou mesmo em compostos orgânicos exibindo cargas negativas residuais, é bastante variável. Resíduos contendo açúcares, amidos e ácidos orgânicos mais simples são rapidamente oxidados no solo, pouco contribuindo para a formação de húmus e consequente aumento da CTC. Já aqueles contendo moléculas orgânicas maiores e/ou mais complexas sofrem oxidação mais lenta e são capazes de contribuir para a formação de húmus, porém dentro de períodos bastante variável, em função dos componentes existentes no resíduo (MELO et al., 2008).

De acordo com Glória (2004), resíduos celulósicos têm mostrado mais "persistência" nos solos, evidenciando que, nas condições de solos tropicais, há necessidade de uma maior atenção na "qualificação" do material orgânico, no sentido da sua real capacidade de provocar alterações mais permanentes na CTC.

Decorre daí a necessidade de estudos específicos em relação à capacidade de os resíduos orgânicos provocarem alterações sensíveis na CTC, vinculada a uma melhor caracterização do seu material orgânico, o que não pode ser feito nas condições atuais e usuais de caracterização química dos resíduos.

\section{Teor de nutrientes}

Para Glória (2004), a utilização dos resíduos no solo pode ser apreciada de duas formas distintas:

a) Utilizando o solo apenas como depurador da carga orgânica;

b) Visando à depuração do resíduo e do aproveitamento dos nutrientes nele contidos.

Nos dois casos, junto com a carga orgânica, estão sendo levados nutrientes. No primeiro caso, apesar do aproveitamento desses nutrientes não estar sendo considerado, é preciso lembrar que alguns desses elementos podem também causar problemas se a taxa de aplicação for elevada. O caso típico é do nitrogênio. Ele pode aparecer nos resíduos em diferentes formas e, uma vez colocado no solo, estará sujeito a reações que conduzem:

$\checkmark$ à imobilização parcial pela incorporação pelos microrganismos do solo e transformação em compostos orgânicos contendo N, mais persistentes no solo;

$\checkmark$ a perdas por volatilização e por lixiviação.

São as perdas por lixiviação as que mais preocupam, pois o destino final do $\mathrm{N}$ adicionado ao solo, em condições aeróbicas, é o nitrato. Esse ânion percola rapidamente nos solos e pode atingir o lençol freático, e o nitrato é nocivo à saúde (FULLER e WARRICK, 1985).

A recomendação da taxa de aplicação de um resíduo, em relação ao $\mathrm{N}$ disponível nele contido, é que a quantidade do resíduo seja correspondente ao nitrogênio que a cobertura vegetal utiliza. Essa quantidade pode ser 50\% maior se a aplicação for 
feita superficialmente, devido às perdas por volatilização que ocorrem (MALAVOLTA, 2006).

Deve ser notado que existe um efeito cumulativo das aplicações de resíduo contendo $\mathrm{N}$, e este efeito é relevante quando se calcula a taxa de aplicação. $\mathrm{Na}$ Tabela 3, está um exemplo da liberação do nitrogênio em função do seu teor no lodo e do período transcorrido após a aplicação.

Tabela 3. Liberação de $\mathrm{N}$ durante a decomposição do lodo no Solo ${ }^{(1)}$.

\begin{tabular}{|c|c|c|c|c|}
\hline \multirow{2}{*}{$\begin{array}{c}\text { Áreas após a } \\
\text { aplicação }\end{array}$} & \multicolumn{4}{|c|}{ Conteúdo de N orgânico no lodo (\%) } \\
\hline & 2,0 & $\mathbf{3 , 0}$ & $\mathbf{4 , 0}$ & $\mathbf{5 , 0}$ \\
\hline & \multicolumn{4}{|c|}{-------------- kg N liberado por t de lodo adicionado ------------- } \\
\hline 1 & 0,50 & 0,70 & 0,95 & 1,20 \\
\hline 2 & 0,45 & 0,70 & 0,90 & 1,15 \\
\hline 3 & 0,45 & 0,85 & 0,85 & 1,10 \\
\hline
\end{tabular}

(1) FULLER e WARRICK (1985).

Obviamente, é importante conhecer os diferentes parâmetros que procuram definir a taxa de aplicação, incluindo-se aí o que se deve ser considerado como "Ndisponível" do resíduo e o efeito do tempo na liberação do N.

Ainda em relação aos nutrientes e à taxa de aplicação do resíduo, devem-se examinar todos os nutrientes nele contidos, procurando estabelecer uma taxa de aplicação em função de um nutriente específico contido em maior porcentagem e, uma vez atendidas às eventuais limitações, dos aspectos de carga orgânica, teor de sais e sódio e dos metais pesados (NOBILE, 2002).

\section{Sais e sódio}

A taxa de aplicação de umresíduo ao solo pode ser limitada, em razão da presença de uma excessiva carga salina. Aavaliação da carga salina e seus efeitos no solo ou nas culturas deve ser em decorrência das condições climáticas da região e da textura e permeabilidade do solo. Regiões áridas e/ou solos argilosos tendem a ter condições mais restritivas ao uso de resíduos salinos (condutividade acima de 2,0 mmnhos $\mathrm{cm}^{-1}$ ), o que corresponde a aproximadamente 1250 ppm de sólidos dissolvidos. Entretanto, em determinadas condições climáticas, requer avaliações específicas (NOBILE, 2005).

Dentre os sais solúveis, nos resíduos é frequente a presença de sódio. Este íon provoca, através do deslocamento do Ca e Mg dos colóides do solo, a dispersão das argilas e consequente perda da condutividade hidráulica do solo. Admite-se que este efeito surge toda vez que o $\mathrm{Na}^{+}$aparece em níveis iguais ou superiores a $15 \%$ do complexo sortivo do solo (NOBILE et al., 2007). 
Uma avaliação do potencial nocivo da sodicidade de um resíduo pode ser feita pela relação de adsorção de sódio (RAS) - de 5 a 15 pode conduzir a problemas de destruição da estrutura dos solos. A RAS é calculada por (BOHNEN et al., 2000):

$$
R A S=\frac{N a}{\sqrt{\frac{C a+M g}{2}}}
$$

onde $\mathrm{Na}, \mathrm{Ca}$ e $\mathrm{Mg}$ indicam a concentração respectiva dos íons sódio, cálcio e magnésio em e.mg L-1 (equivalente miligrama por litro) de solução.

Outra vez se deve ficar alerta acerca dos nossos poucos conhecimentos a respeito desses índices, em condições de clima e solo, principalmente das Regiões Central, Sul e Sudeste do país.

\section{Metais pesados}

Para Glória (2004), uma das grandes preocupações, quando se cogita a aplicação de resíduos no solo, é o seu conteúdo de metais pesados, incluindo-se nessa discussão elementos de densidade inferior a 5,0, caso do boro.

No tocante aos metais pesados, suas presenças em níveis excessivos podem inviabilizar a aplicação no solo ou exigir pré-tratamentos para sua redução e posterior uso (NOBILE et al., 2008).

A quantidade de resíduo a aplicar, em função dos seus teores em metais pesados, é dependente de uma série de propriedades dos solos, entre as quais se podem citar: pH, CTC, teor de argila, óxidos de Fe, Ale Mn, carbono orgânico total (COT) e sólidos solúveis (FULLER e WARRICK, 1985).

A preocupação com o nível de metais pesados advém da capacidade de retenção dos mesmos pelo solo, sua movimentação no perfil do solo e possibilidade de atingir o lençol freático e a biodisponibilidade para os diferentes vegetais.

Os mecanismos que auxiliam sua retenção via precipitação e ou absorção são: pH, CTC, teor de argila e de óxidos hidratados de ferro. Quanto maiores esses valores, maior a possibilidade de retenção. A presença de matéria orgânica, sais solúveis ou condições anaeróbicas facilitam a movimentação no perfil e favorecem a absorção pelos vegetais (SOUZA et al., 2005).

A Tabela 4 exemplifica as recomendações vigentes nos Estados Unidos, quanto à adição de metais pesados e à CTC dos solos. 
Tabela 4. Quantidade total de metais permitida em áreas agrícolas ${ }^{(1)}$

\begin{tabular}{cccc}
\hline \multirow{2}{*}{ Metais } & \multicolumn{3}{c}{ CTC do solo $\left(\mathrm{cmol}_{\mathrm{c}} \mathrm{dm}^{-3}\right)$} \\
\cline { 2 - 4 } & $0-5$ & $6-15$ & $>15$ \\
\hline $\mathrm{PB}$ & 600 & 1200 & 2400 \\
$\mathrm{Zn}$ & 300 & 600 & 1200 \\
$\mathrm{Cu}$ & 150 & 300 & 600 \\
$\mathrm{Ni}$ & 150 & 300 & 600 \\
$\mathrm{Cd}$ & 6 & 12 & 24 \\
\hline
\end{tabular}

${ }^{(1)}$ Adaptada de Fuller e Warrick (1985).

${ }^{(2)}$ Quantidade total que pode ser aplicada, em uma ou mais aplicações, sempre mantendo o $\mathrm{pH}$ do solo $(6,5)$

$\mathrm{cmol}_{\mathrm{c}} \mathrm{dm}^{-3}$ (centimol de carga por decímetro cúbico)

É óbvio que o conhecimento da composição química do resíduo e dos locais disponíveis para a sua aplicação demonstrarão como esta deve ser definida, para que se elimine o perigo de contaminações e possibilite a utilização mais prolongada possível das áreas de disposição.

\section{Matéria orgânica}

A avaliação da matéria orgânica dos resíduos pode ser feita por meio de diferentes determinações: sólidos solúveis, oxigênio consumido (OC), demanda química de oxigênio (DQO), demanda biológica de oxigênio (DBO).

Os valores dessas determinações não são iguais, mas as suas correlações são muito altas e, inquestionavelmente, quanto mais altos os seus valores maior o conteúdo de matéria orgânica do resíduo. Admite-se que a forma mais adequada seria a determinação da DQO, reservando-se a DBO para avaliação em efeitos nos cursos d'água, uma vez que a ação microbiana no solo é mais diversificada e dinâmica que nas águas (GLÓRIA, 2004).

Provavelmente, o maior interesse do uso dos resíduos no solo reside na capacidade deste meio em decompor a matéria orgânica, sem afetar o ambiente de forma significativa.

A taxa de aplicação de um resíduo ao solo deve prever, portanto, que o solo será capaz de agir para decompor a matéria orgânica, sem riscos de lixiviação pelo perfil do solo - ou de transporte - por processos erosivos - para coleções hídricas, e ainda sem causar odores ou perturbações de ordem estética e social (SANTOS e TOMM, 2003).

Entretanto, os resíduos apresentam uma grande diversidade de compostos orgânicos, os quais, por sua vez, têm diferentes taxas de decomposição no solo. A Figura 2 procura demonstrar isso. 


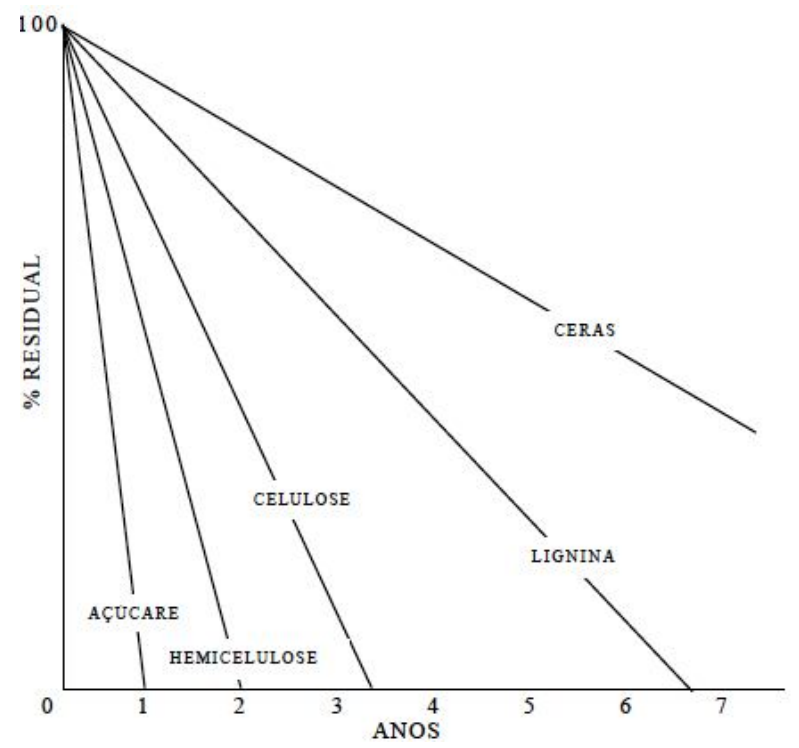

Figura 2. Curvas de decomposição para vários constituintes orgânicos (FULLER e WARRICK, 1986).

Além disso, a presença de outros nutrientes, notadamente o nitrogênio e o fósforo, afetam pronunciadamente a decomposição da matéria orgânica. $O$ consumo de nitrogênio pela decomposição da matéria orgânica é avaliado em 5\% da DBO. Assim, se o resíduo tem DBO de 800 ppm e a vazão é de $50 \mathrm{~L} / \mathrm{s}$, serão necessários $4147 \mathrm{~kg}$ de nitrogênio mensais, a fim de que a remoção de DBO atinja $80 \%$. Sendo que o nitrogênio a considerar é o nitrogênio inorgânico e não o nitrogênio total (FULLER e WARRICK, 1986).

Em relação ao fósforo, a boa remoção da DBO é obtida quando a relação DBO/ fósforo é inferior a 100. Portanto, com um resíduo de 800 ppm de DBO, no mínimo deve-se ter 8 ppm de fósforo, para uma boa remoção da carga orgânica. É óbvio que, quando se cogita a aplicação no solo, esse substrato já pode conter o fósforo mínimo necessário para a remoção, ou então, se o resíduo for pobre no elemento, este deverá ser adicionado (FULLER e WARRICK, 1986).

A condição de decomposição aeróbica é mais eficiente que a anaeróbica para a remoção da matéria orgânica. Portanto, se através de uma adição excessiva de resíduos se conduzir a uma condição anaeróbica, reduz-se a taxa de remoção e perde-se eficiência do sistema.

A pré-caracterização do resíduo indica a possibilidade de compostos orgânicos desse tipo, e as suas presenças exigirão não só a determinação específica, mas 
também o estudo em condições de laboratório e campo, da sua degradabilidade, movimento no solo e potenciais de riscos com outros testes, como os de toxidez e mutagenicidade.

\section{Procedimentos para o uso de resíduos no solo}

Procurou-se evidenciar que a utilização de resíduos no solo pode e deve ser praticada, mediante cuidados especiais que compreendam várias etapas, que vão da pré-caracterização dos resíduos na sua origem aos estudos de seus efeitos específicos no solo.

Na Figura 3 está um fluxograma dos procedimentos que são recomendados (GLÓRIA, 2004).

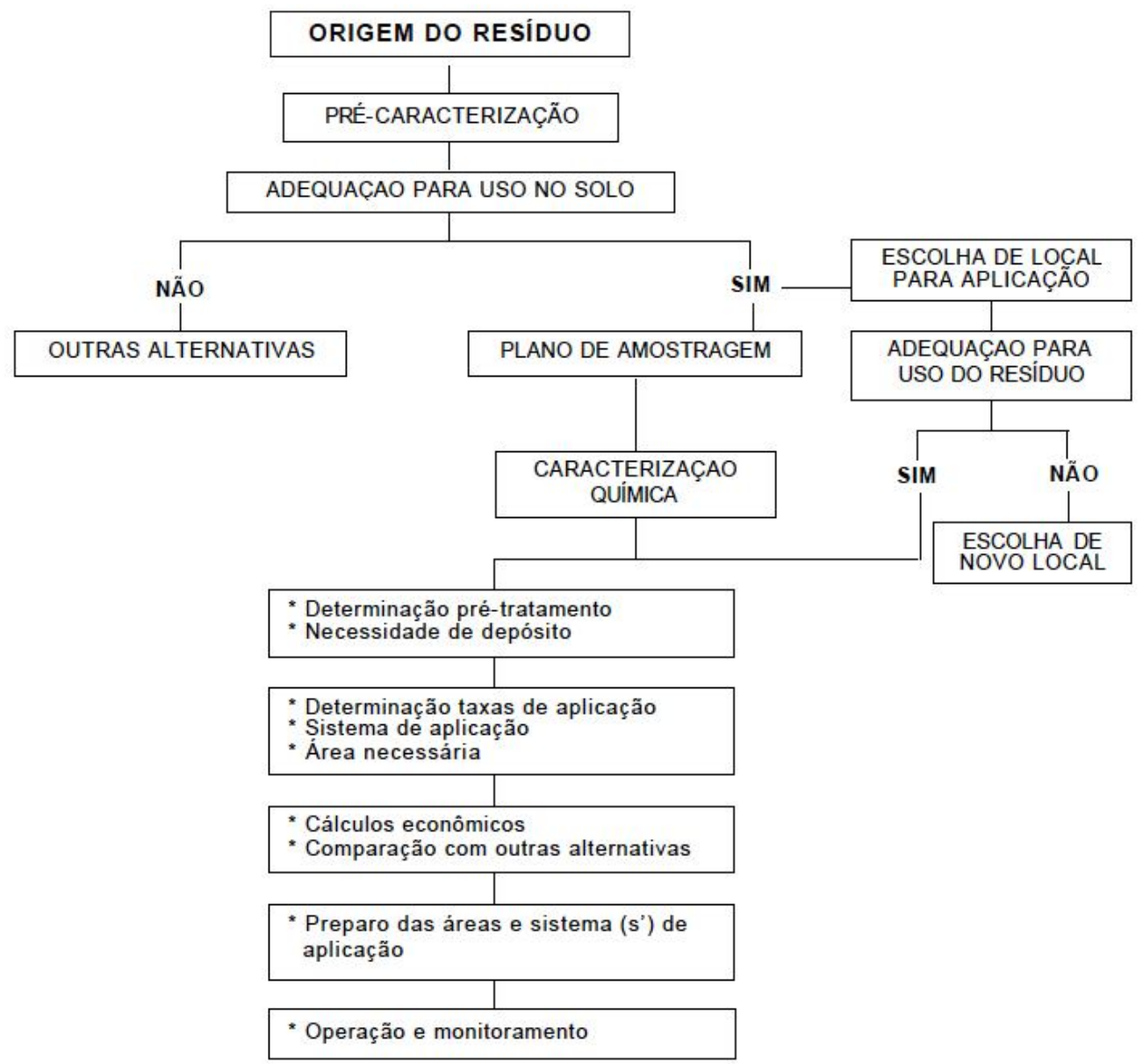

Figura 3. Fluxograma dos procedimentos para uso de resíduos no solo (GLÓRIA, 2004). 
Deve ser notado que, além dos aspectos já tratados nos itens precedentes, os procedimentos para uso do resíduo no solo não se esgotam na sua caracterização e efeito no solo. A definição sobre a necessidade de pré-tratamentos será baseada na caracterização química, e os efeitos no solo devem ser testados com o resíduo já tratado.

A vazão dos resíduos e sua periodicidade, sazonalidade e homogeneidade vão ditar a necessidade de depósitos, que poderão servir para um armazenamento, em função do sistema de aplicação a ser adotado, e como colaboração para uma maior homogeneidade do resíduo.

As taxas de aplicação estão ligadas ao sistema de aplicação a ser empregado e que, por sua vez, está relacionado à disponibilidade de área, sua localização em relação ao ponto gerador, topografia e aspectos legais e sociais.

Uma vez definidas as possibilidades do uso, taxa de aplicação e sistema a ser adotado é imprescindível o cálculo econômico do que está sendo proposto, principalmente em comparação com alternativas de tratamento. É importante caracterizar os aspectos dos investimentos fixos e variáveis, a fim de se poder ter uma real avaliação das opções. Geralmente, processos alternativos ao uso no solo têm custos variáveis extremamente elevados pelo gasto com energia e produtos químicos, enquanto os de aplicação se caracterizam pelos elevados custos do transporte do resíduo. Já em relação aos investimentos fixos, os sistemas de uso no solo têm a vantagem de, muitas vezes, não exigir grandes dispêndios, enquanto os processos alternativos em geral exigem investimentos iniciais elevados.

Complementarmente, deve ser lembrado que o uso dos resíduos no solo deve ser conduzido no sentido de não só eliminar a sua nocividade, mas também tornar atraente sua utilização como fonte de nutrientes para as culturas, ou como condicionador do solo. Nesses casos é possível que o resíduo se torne um subproduto, passando a ter um valor de comércio.

Uma vez decidida a implantação do sistema, passa-se às fases de execução e operacionais, compreendidas pelo preparo das áreas e do funcionamento do sistema de aplicação. Já a operacionalização não deve compreender apenas o transporte e aplicação, sendo fundamental o acompanhamento do processo, por meio do monitoramento das condições do solo e das águas subterrâneas e/ou adjacentes ao local(is) de aplicação.

\section{Conclusão}

A aplicação de resíduos no solo abre perspectivas de estudos bastante amplos e reconhece-se a extrema carência de dados, de climas e de solos sobre os efeitos de seu uso. O que se espera é a conscientização para o problema e o apoio para que se 
formem especialistas nessas áreas, por meio, principalmente, de pesquisas sérias e objetivas sobre todos os aspectos aqui abordados e aqueles que não foram considerados, devido à extensão do assunto.

\section{REFERÊNCIAS}

BOHN H. L.; McNEAL B. L.; O'CONNOR G.A. Soil Chemistry. USA, John Wiley \& sons, inc, 1985. 341 p.

DEFELIPO, B. V.; NOGUEIRA, A. V.; LOURDES, E .G.; ALVAREZ, V. V. H. Eficiência agronômica de um resíduo de indústria siderúrgica. Revista Brasileira de Ciência do Solo, Campinas, v.16, n.1, p.127-131, 1992.

FALCÃO, A. de A. Análise química de resíduos sólidos para estudos agroambientais. 2005. Dissertação (Mestrado em Química Analítica) Universidade Estadual de Campinas, Instituto de Química, Campinas, 2005.

FULLER, W. H.; WARRICK, A. W. Soils in waste treatment and utilization. Boca Raton: CRC Press, 1985. v.1, 268p.

GLÓRIA, N. A. Uso Agronômico de resíduos. In: REUNIÃO BRASILEIRADE FERTILIDADE DO SOLO E NUTRIÇÃO DE PLANTAS, 20, 2004, Lages. Anais... Lages: SBCS, 2004. 1 CD-ROM.

GLÓRIA, N. A.; MATTIAZZO, M. E. Studies on land disposal of furfural production effluent. In: International Environmental Chemistry Congress, 1991, Salvador. Abstract of 3 International Environmental Chemistry Congress. p. 50-50.

MALAVAOLTA, E. Manual da nutrição mineral de plantas. Piracicaba: Ceres, 2006. 638 p.

MATTIAZZO, M. E.; GLORIA, N. A. Efeito da vinhaça na acidez do solo. STAB-Açúcar, Álcool e Subprodutos, v.4, n.2, p.35-40, 1985.

MELO, L. C. A.; SILVA, C. A.; DIAS, B.O. Caracterização da matriz orgânica de resíduo de origens diversificadas. Revista Brasileira de Ciência do Solo, v. 32, p.101-110, 2008. 
NOBILE, F. O. de. Variáveis agro-industriais da cana-de-açúcar cultivada em solo fertilizado com lodo de esgoto e vinhaça. 2002. 67p. Monografia (Trabalho de Graduação em Agronomia) Faculdade de Ciências Agrárias e Veterinárias, Universidade Estadual Paulista, Jaboticabal, 2002.

NOBILE, F. O. de. Efeito da aplicação de resíduo da mineração de bauxita no solo e na planta de cana-de-açúcar. 2005. 117f. Dissertação (Mestrado em Ciência do Solo) Faculdade de Ciências Agrárias e Veterinárias, Universidade Estadual Paulista, Jaboticabal, 2005.

NOBILE, F. O. de; GALBIATTI, J. A.; MURAISHI, R. I.; RIBEIRO, A. G. Sódio em solo adubado com fertilizantes minerais e orgânicos e irrigado com água servida. In: CONGRESSO BRASILEIRO DE ENGENHARIAAGRÍCOLA, 36, 2007, Bonito. Anais... Bonito: SBEA, 2007. 1 CD-ROM.

NOBILE, F. O. de; GALBIATTI, J. A.; MURAISHI, R. I. Contamination of seepage water in lettuce crop superficially irrigated with water containing urban sewage and treated water in green house In: CONGRESSO BRASILEIRO DE ENGENHARIAAGRÍCOLA, 37., 2008, Rio de Janeiro. Anais... Rio de Janeiro: SBEA, 2008. 1 CD-ROM.

PERUCCI, P. Effect of the addition of municipal solid-waste compost on microbial biomass and enzyme avtivities in soil. Biol. Fertil. Soils, Berlin, v.10, p.221-226, 1990.

RODELLA, A. A.; FERRARI, S. E. . Ocorrência de dextrana em cana-deaçúcar. Brasil Açucareiro, Rio de Janeiro, v. 96, n. 5, p. 380-387, 1977.

\section{SANTOS, B. D. Dinâmica de enxofre no sistema solo e resposta das} culturas à adubação sulfatada. 2006. Dissertação (Mestrado em Ciência do Solo) - Universidade Federal de Santa Maria, Santa Maria, 2006.

SANTOS, H. P.; TOMM, G. O. Disponibilidade de nutrientes e teor de matéria orgânica função de sistemas de cultivo e de manejo de solo. Ciência Rural, Santa Maria, v.33, n.3, p.477-486, 2003.

SOUZA, R. M. P; LEAO, V. A.; PINA, P. dos S.s. Remoção de metais pesados em resíduos sólidos: o caso das baterias de celular. Rem: Rev. Esc. 
Minas, Ouro Preto, v. 58, n. 4, 2005.

VELOSO, C. A. C.; BORGES, A. L.; MUNIZ, A. S.; VEIGAS, I. A. de J. M. Efeito de diferentes materiais no ph do solo. Scientia Agrícola, v. 49, n. 1, p. 123-128, 1992.

\section{TITLE: USE OF RESIDUES IN AGRICULTURE}

\section{ABSTRACT}

This article presents a revision of the abstracts of The XX Brazilian Meeting of Fertility of the Soil and Nutrition of Plants, realizado in Piracicaba-São Paulo. The agronomic use of residues pressuposes a diverse number of applications such as their use in: animal feeding, substrate for fermentations, organic or organo-mineral fertilizer manufacture, soil coverages in different creations and substitutes of raw material for the agroindustrial or similar activities. However, the objective of this presentation is restricted to the aspects of the use of residues in the soil, understanding the aspects related to the characterization of these residues, the benefits or inconveniences of the application to the soil and the parameters that must be observed when the intention is to give this destination to the residues. In this way, this subject can be divided in two basic aspects: the residues and the soils. Concerning the residues, the main factors that affect their application to the soil are: chemical composition, physical characteristics, sanitary aspects, generated amount and regimen of release. In relation to soil, we must consider all those characteristics which are responsible for the capacity of the soil in deactivating and stabilizing the residues, through physical chemical and biological mechanisms. It is in this general framework that we intend to approach some specific aspects which are considered more important for the use of residues in soil.

KEYWORDS: Agriculture; Residues; Soil. 